\title{
A Single-Photon Router in Quantum Fluctuation of Field
}

\author{
LI-Wei LiU ${ }^{a}, b, *$, YU-QING Shi ${ }^{a}$ AND Chen QIONG ${ }^{a}$ \\ ${ }^{a}$ College of Electrical Engineering, Northwest University for Nationalities, Lanzhou 730000, China \\ ${ }^{b}$ Key Laboratory for Electronic Materials of the State Ethnic Affairs Commission of PRC, \\ Northwest University for Nationalities, Lanzhou 730000, China
}

(Received 25 February 2017; in final form 14 July, 2017)

We investigate a single-photon quantum router in quantum fluctuation of fields. The optomechanical system composed of a cigar-shaped Bose-Einstein condensate trapped in an ultrahigh-finesse Fabry-Pérot cavity. We show how an analog of electromagnetically induced transparency in an optomechanical system can be used to produce a switch for a quantum fluctuation field using very low pumping field strength. The numerical results show that the output photon is completely different by turning the pump off and turning the pump on. We also show that the quantum noise sources are very small. This optomechanical system can serve as a single-photon quantum router.

DOI: 10.12693/APhysPolA.132.1358

PACS/topics: single-photon quantum router, Bose-Einstein condensate, cavity optomechanical

\section{Introduction}

In recent years, much research has been conducted in the field of quantum information science [1,2]. Photons can carry quantum information like electrons [3-5]. There are many advantages using photon-transmitted information. For example, it is possible to distribute information over long distances without much decoherence. The method can also provide strong interactions between radiation and matter as photons that do not interact by themselves. In addition, photon-transmitted information can propagate fast and interact with the environment rarely. Many quantum information protocols such as quantum cryptography [6] and quantum networks [7] require single-photon. A single-photon quantum router plays a key role in quantum information science [8-13]. Single-photon routers receive high interests by both applied researchers [14, 15] and theoreticians [16]. Several proposals have been made for the realization of a single-photon router. In an early study, atomic electromagnetically induced transparency (EIT) in a cavity has been used to operate a single-photon router [17]. Furthermore, a single atom in a strongly coupled waveguide array and atoms via surface plasmons on a nanowire were used to realize a single-photon router [18-21]. There are also reports of a single-photon router based on a coupled cavity optomechanical systems, such as nanomechanics mirrors and BEC in optical cavities [22, 23]. In this study, we show how a Bose-Einstein condensate(BEC)optomechanical system can be used to build a singlephoton quantum router.

A BEC-optomechanical system, which consists of a BEC trapped inside an optical cavity, has been proposed and attracted much attention [24-30]. For such a system, the intra-cavity laser field excites a momentum side-mode of the condensate [24-26]. Furthermore, in the BEC

*corresponding author; e-mail: liuliw@xbmu.edu.cn optomechanical system, a strong coupling range can be reached easily even with an ultra-low pump power [2730]. More importantly, the BEC can be trapped on a small scale, and thus a robust miniature device can be implemented easily [31-33]. The BEC-optomechanical system [22], which is fed by both a detuned strong pump field and a weak probe field, can serve as a single photon router. Now we will study the quantum fluctuation of the cavity without any input probe field, the BECoptomechanical system can be used to produce a switch. The quantum fluctuation of the fields arises directly from the fluctuation of the vacuum input and the fluctuation of the effective of the mechanical oscillator mode. Therefore, the BEC-optomechanical system is a promising candidate to build a quantum single-photon router.

The paper is structured as follows. In Sect. 2, we derive the Hamiltonian of the optomechanical systems consisting of a BEC trapped inside an optical cavity. The reflection and transmission of the output field are obtained in this section. In Sect. 3, the numerical results show that the output photon changes completely different by turning the pump off and turning the pump on. We also show that the quantum noise sources of this phenomenon are very small. A summary is presented in Sect. 4.

\section{The physical model}

We consider an optomechanical system with a cigarshaped BEC trapped in an ultrahigh-finesse Fabry-Pérot cavity of length $L$, which is formed by two fixed mirrors with finite identical transmission, see Fig. 1. We denote the cavity field by $\hat{c}$, frequency $\omega_{c}$, decay rate $\kappa, \hat{c}_{i n}$ and $\hat{d}_{i n}$ are the quantum fields. We give the Hamiltonian of the optomechanical systems consisting of a BEC trapped inside an optical cavity as

$$
\begin{aligned}
\hat{H} & =\hbar \omega_{c} \hat{c}^{\dagger} \hat{c}-\mathrm{i} \hbar E_{p}\left(\hat{c} \mathrm{e}^{\mathrm{i} \omega_{p} t}-\hat{c}^{\dagger} \mathrm{e}^{-\mathrm{i} \omega_{p} t}\right) \\
& +\int_{0}^{L} \mathrm{~d} x \Psi^{\dagger}(x)\left[\frac{-\hbar^{2}}{2 m_{a}} \frac{\mathrm{d}^{2}}{\mathrm{~d} x^{2}}+V_{e x t}(x)\right] \Psi(x) \\
& +\int_{0}^{L} \mathrm{~d} x \Psi^{\dagger}(x) \hbar U_{0} \cos ^{2}(k x) \hat{c}^{\dagger} \hat{c} \Psi(x),
\end{aligned}
$$




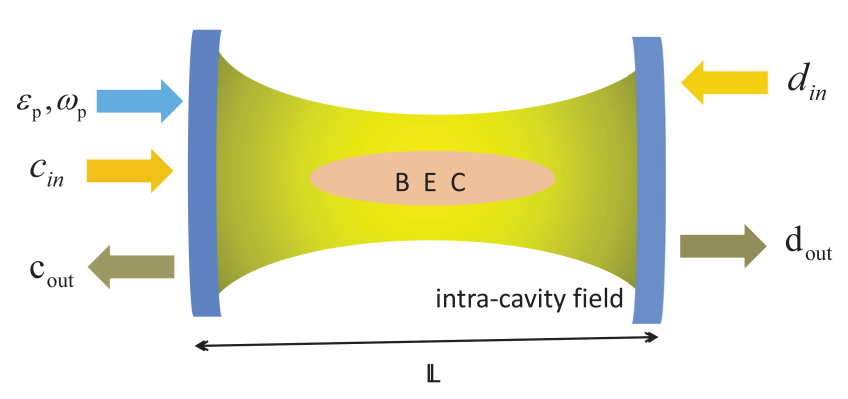

Fig. 1. The optomechanical system consisting of a Bose-Einstein condensate trapped inside an optical cavity. The cavity, which decays at rate $\kappa$, is driven by one laser with frequency $\omega_{p}$.

where the first term describes the energy of the intracavity field, $\omega_{c}$ is the cavity frequency, and $\hat{c}$ is the cavity field's annihilation operator. The second term describes the energy of the pump laser field with strength $E_{p}$, and $E_{p}$ is related to the laser power $P$ via $\left|E_{p}\right|=\sqrt{2 P \kappa / \hbar \omega_{p}}$, and $\kappa$ is the cavity decay rate. The third term describes the Hamiltonian of the condensate of the BEC in the case of avoiding atom-atom interaction and assuming a shallow external trapping potential. Here $\Psi$ is a bosonic field annihilation operator, $m_{a}$ is the atomic mass, $V_{\text {ext }}$ is the external potential. The fourth term describes the energy of the atom-cavity coupling interaction, where $U_{0}=g_{0}^{2} / \Delta_{a}$ is the maximum light shift that an atom experiences in the cavity mode. $\Delta_{a}$ describes the detuning between the pump laser frequency and the atomic transition frequency, $g_{0}$ is the maximum coupling strength between a single atom and a single intracavity photon, $k$ is the wave number of the light field. In our paper, we consider the following strong coupling regime $\left(N g_{0}^{2} /\left|\Delta_{a}\right| \gg \kappa\right.$, where $N$ is the average number of atoms).

We consider the simplest situation where the optical field is weak enough, and only the first two symmetric momentum side modes with moment $\pm 2 \hbar k$ are excited by fluctuations resulting from the atom-light interaction. Considering the parity conservation and the Bogoliubov approximation, the atomic field operator can be expanded as follows:

$$
\Psi(x)=\sqrt{\frac{1}{L}} \hat{a}_{0}+\sqrt{\frac{2}{L}} \cos (2 k x) \hat{a}_{2},
$$

here $\hat{a}_{0}$ and $\hat{a}_{2}$ are bosonic annihilation operators for atoms in the zero-momentum state and side-mode components, respectively. The total number of atoms is $\hat{a}_{0}^{\dagger} \hat{a}_{0}+\hat{a}_{2}^{\dagger} \hat{a}_{2}=N$. Because the population in the zeromomentum state is much larger than the population in the side-mode state, we can neglect the population in the side-mode state. We use $\hat{a}_{0}^{\dagger} \hat{a}_{0} \simeq N$, and $\hat{a}$ instead of $\hat{a}_{2}$. In a rotating frame at driving field frequency $\omega_{p}$, the Hamiltonian of the optomechanical system can be expressed as follows:

$$
\begin{aligned}
\hat{H} & =\hbar \Delta_{c} \hat{c}^{\dagger} \hat{c}-\mathrm{i} \hbar E_{p}\left(\hat{c}-\hat{c}^{\dagger}\right)+\hbar \omega_{m} \hat{a}^{\dagger} \hat{a} \\
& +\hbar g\left(\hat{a}^{\dagger}+\hat{a}\right) \hat{c}^{\dagger} \hat{c},
\end{aligned}
$$

where $\omega_{m}=4 \omega_{r e c}=4 \frac{\hbar k^{2}}{2 m}, \Delta_{c}=\omega_{c}^{\prime}-\omega_{p}, \omega_{c}^{\prime}=$ $\omega_{c}+\frac{1}{2} U_{0} N$, and $g=\frac{U_{0}}{2} \sqrt{\frac{N}{2}}$. The first term is the energy of the intra-cavity field, $\Delta_{c}$ is the effective Starkshift detuning, $\frac{1}{2} U_{0} N$ denote the frequency shift of the empty cavity resonance induced by the BEC. The second term represents the energy of the driving field. The third term is the energy of the Bogoliubov mode of the collective oscillation of the BEC, where $\hat{a}\left(\hat{a}^{\dagger}\right)$ denote the annihilation (creation) operator of the Bogoliubov mode. The last term describes the coupling energy between the BEC and the cavity, where $g$ is the coupling strength.

The motion of BEC in the cavity is formally analogous to the harmonic oscillator. We define the position operator $\hat{X}=\frac{1}{\sqrt{2}}\left(\hat{a}+\hat{a}^{\dagger}\right)$, and the momentum operator $\hat{Y}=\frac{i}{\sqrt{2}}\left(\hat{a}^{\dagger}-\hat{a}\right)$. The commutation relation is $[\hat{X}, \hat{Y}]=i$. According to the commutation relation $\left[\hat{a}, \hat{a}^{\dagger}\right]=1$, and $\left[\hat{c}, \hat{c}^{\dagger}\right]=1$, we add up all the quantum fields, thermal fluctuations, and the Hamiltonian from Eq. (3), the coupled quantum Langevin equations for $\hat{c}, \hat{X}$ and $\hat{Y}$ can be obtained as

$$
\begin{aligned}
& \frac{\mathrm{d} \hat{c}}{\mathrm{~d} t}=-\left(\mathrm{i} \Delta_{c}+2 \kappa+\mathrm{i} g \hat{X}\right) \hat{c}+E_{p}+\sqrt{2 \kappa} \hat{c}_{i n}+\sqrt{2 \kappa} \hat{d}_{i n}, \\
& \frac{\mathrm{d} \hat{X}}{\mathrm{~d} t}=\omega_{m} \hat{Y}-\gamma_{s m} \hat{X}+\hat{f}_{1 m}, \\
& \frac{\mathrm{d} \hat{Y}}{\mathrm{~d} t}=-\omega_{m} \hat{X}-g \hat{c}^{\dagger} \hat{c}-\gamma_{s m} \hat{Y}+\hat{f}_{2 m} .
\end{aligned}
$$

The corresponding noises operators are $\hat{c}_{i n}, \hat{d}_{i n}, \hat{f}_{1 m}$ and $\hat{f}_{2 m}$. Noise operators depends on the reservoir variables. On the one hand, we have introduced the input vacuum noise operators $\hat{c}_{i n}, \hat{d}_{i n}$ with the zero average values, which satisfies the Markovian correlation functions

$$
\begin{aligned}
\left\langle\hat{x}_{i n}(t) \hat{x}_{i n}^{\dagger}\left(t^{\prime}\right)\right\rangle & =2 \kappa\left(n_{p}+1\right) \delta\left(t-t^{\prime}\right), \\
\left\langle\hat{x}_{i n}^{\dagger}\left(t^{\prime}\right) \hat{x}_{i n}(t)\right\rangle & =2 \kappa n_{p} \delta\left(t-t^{\prime}\right),
\end{aligned}
$$

where $x=\hat{c}, \hat{d}$, the cavity field $\hat{c}$ is coupled to the input quantum field $\hat{c}_{i n}$ and $\hat{d}_{i n}$, with the average thermal photon number $n_{p}$, which is nearly zero at cavity frequency [34]. $\delta\left(t-t^{\prime}\right)$ is the Dirac delta function.

On the other hand, $\hat{f}_{1 m}$ and $\hat{f}_{2 m}$ are the thermal noise inputs for the effective of the mechanical oscillator mode of BEC, which also satisfy the same Markovian correlation functions as those of the cavity noise

$$
\begin{aligned}
& \left\langle\hat{f}_{1 m}(t) \hat{f}_{1 m}\left(t^{\prime}\right)\right\rangle=\gamma_{s m} \delta\left(t-t^{\prime}\right), \\
& \left\langle\hat{f}_{2 m}(t) \hat{f}_{2 m}\left(t^{\prime}\right)\right\rangle=\gamma_{s m} \delta\left(t-t^{\prime}\right) .
\end{aligned}
$$

We want to calculate the spectrum of the output field by using the quantum Langevin Eqs. (4) and following standard methods from quantum optics [35]. First, we linearize the quantum Langevin equations by making the ansatz as follow $\hat{O}=O_{s}+\delta \hat{O}$ (with $\left.O \in\{\hat{c}, \hat{X}, \hat{Y}\}\right)$. $O_{s}$ is the steady-state mean value of the observable and $\delta \hat{O}$ is small fluctuation. We derive steady-state mean values by setting all the time derivatives in Eq. (4) to zero, the steady values are given as 


$$
c_{s}=\frac{E_{p}}{2 \kappa+\mathrm{i} \Delta}, \quad X_{s}=\frac{-g\left|c_{s}\right|^{2}}{\omega_{m}\left(1+\frac{\gamma_{s m}^{2}}{\omega_{m}^{2}}\right)}, \quad Y_{s}=\frac{\gamma_{s m} X_{s}}{\omega_{m}},
$$

with $\Delta=\Delta_{c}-\frac{g^{2}\left|c_{s}\right|^{2}}{\omega_{m}\left(1+\frac{\gamma_{s m}^{2}}{\omega_{m}^{2}}\right)}, c_{s}$ denotes the steady state amplitude of the cavity field. $X_{s}\left(Q_{s}\right)$ represents the new equilibrium position (momentum) of the collective oscillation of the BEC.

The linearized quantum Langevin equations for the fluctuation operator are as follows:

$$
\begin{aligned}
& \frac{\mathrm{d} \delta \hat{c}}{\mathrm{~d} t}=-(\mathrm{i} \Delta+2 \kappa) \delta \hat{c}-\mathrm{i} g c_{s} \delta \hat{X}+\sqrt{2 \kappa} \hat{c}_{i n}+\sqrt{2 \kappa} \hat{d}_{i n}, \\
& \frac{\mathrm{d} \delta \hat{X}}{\mathrm{~d} t}=\omega_{m} \delta \hat{Y}-\gamma_{s m} \delta \hat{X}+\hat{f}_{1 m}, \\
& \frac{\mathrm{d} \delta \hat{Y}}{\mathrm{~d} t}=-\omega_{m} \delta \hat{X}-g\left(c_{s} \hat{c}^{\dagger}+c_{s}{ }^{*} \hat{c}\right)-\gamma_{s m} \delta \hat{Y}+\hat{f}_{2 m} .(8)
\end{aligned}
$$

Then, we use the Fourier transforms for these equations $f(t)=\frac{1}{2 \pi} \int_{-\infty}^{\infty} f(\omega) \mathrm{e}^{-\mathrm{i} \omega t} \mathrm{~d} \omega, \quad f^{\dagger}(t)=$ $\frac{1}{2 \pi} \int_{-\infty}^{\infty} f^{\dagger}(-\omega) \mathrm{e}^{-\mathrm{i} \omega t} \mathrm{~d} \omega$ for any operator $f(t) \quad \in$ $\{\hat{c}, \hat{X}, \hat{Y}\}$. Equation (8) can be written as

$$
\begin{aligned}
& -\mathrm{i} \omega \delta \hat{c}(\omega)=-(\mathrm{i} \Delta+2 \kappa) \delta \hat{c}(\omega)-\mathrm{i} g c_{s} \delta \hat{X}(\omega) \\
& +\sqrt{2 \kappa} \hat{c}_{i n}(\omega)+\sqrt{2 \kappa} \hat{d}_{i n}(\omega), \\
& -\mathrm{i} \omega \delta \hat{X}(\omega)=\omega_{m} \delta \hat{Y}(\omega)-\gamma_{s m} \delta \hat{X}(\omega)+\hat{f}_{1 m}(\omega), \\
& -\mathrm{i} \omega \delta \hat{Y}(\omega)=-\omega_{m} \delta \hat{X}(\omega)-g\left(c_{s} \hat{c}^{\dagger}(-\omega)+c_{s}{ }^{*} \hat{c}(\omega)\right) \\
& -\gamma_{s m} \delta \hat{Y}(\omega)+\hat{f}_{2 m}(\omega) .
\end{aligned}
$$

The corresponding noises operators $\hat{c}_{i n}, \hat{d}_{i n}, \hat{f}_{1 m}$ and $\hat{f}_{2 m}$ satisfy the following correlation function:

$$
\begin{aligned}
& \left\langle\hat{x}_{i n}(\omega) \hat{x}_{i n}^{\dagger}(-\omega)\right\rangle=1, \\
& \left\langle\hat{f}_{1 m}(\omega) \hat{f}_{1 m}(-\omega)\right\rangle=\gamma_{s m}, \\
& \left\langle\hat{f}_{2 m}(\omega) \hat{f}_{2 m}(-\omega)\right\rangle=\gamma_{s m},
\end{aligned}
$$

and the solution of $\delta \hat{c}(\omega)$ :

$$
\begin{gathered}
\delta \hat{c}(\omega)=E(\omega)\left(\hat{c}_{i n}(\omega)+\hat{d}_{i n}(\omega)\right)+F(\omega)\left(\hat{c}_{i n}^{\dagger}(-\omega)\right. \\
\left.+\hat{d}_{i n}^{\dagger}(-\omega)\right)+V_{1}(\omega) \hat{f}_{1 m}(\omega)+V_{2}(\omega) \hat{f}_{2 m}(\omega),
\end{gathered}
$$

in which

$$
\begin{aligned}
E(\omega) & =\frac{\sqrt{2 \kappa}}{(2 \kappa+\mathrm{i} \Delta-\mathrm{i} \omega)}+\frac{\sqrt{2 \kappa} i g^{2}\left|c_{s}\right|^{2}(2 \kappa-\mathrm{i} \Delta-\mathrm{i} \omega)}{d(\omega)} \\
F(\omega) & =\frac{\mathrm{i} \sqrt{2 \kappa} g^{2} c_{s}^{2}}{d(\omega)} \\
V_{1}(\omega) & =\frac{-\mathrm{i} \sqrt{2 \kappa} g c_{s}(2 \kappa-\mathrm{i} \Delta-\mathrm{i} \omega)\left(\gamma_{s m}-\mathrm{i} \omega\right)}{d(\omega) \omega_{m}} \\
V_{2}(\omega) & =\frac{-\mathrm{i} \sqrt{2 \kappa} g c_{s}(2 \kappa-\mathrm{i} \Delta-\mathrm{i} \omega)}{d(\omega)}
\end{aligned}
$$

with

$$
\begin{aligned}
& d(\omega)=\frac{\left((2 \kappa-i \omega)^{2}+\Delta^{2}\right)\left(\omega_{m}^{2}+\left(\gamma_{s m}-\mathrm{i} \omega\right)^{2}\right)}{\omega_{m}} \\
& -2 \Delta g^{2}\left|c_{s}\right|^{2} .
\end{aligned}
$$

Then, using the input-output relation $c_{\text {out }}(\omega)=$ $\sqrt{2 \kappa} c(\omega)-c_{i n}(\omega)$, defining the spectrum of the field via $\left\langle\delta \hat{c}^{\dagger}(-\Omega) \delta \hat{c}(\omega)\right\rangle=2 \pi S_{c}(\omega) \delta(\omega+\Omega),\left\langle\delta \hat{c}(-\Omega) \delta \hat{c}^{\dagger}(\omega)\right\rangle=$ $2 \pi\left(S_{c}(\omega)+1\right) \delta(\omega+\Omega)$. The incoming vacuum field $d_{i n}$ is $\left\langle\delta \hat{d}^{\dagger}(-\Omega) \delta \hat{d}(\omega)\right\rangle=2 \pi \delta(\omega+\Omega)$ with $S_{\text {din }}=0$. We can obtain the spectrum of the output fields

$$
\begin{aligned}
& \S_{\text {cout }}(\omega)=R(\omega) S_{\text {cin }}+S^{v}(\omega)+S_{1}(\omega)+S_{2}(\omega), \\
& S_{\text {dout }}(\omega)=T(\omega) S_{\text {cin }}+S^{v}(\omega)+S_{1}(\omega)+S_{2}(\omega) .
\end{aligned}
$$

Here

$$
\begin{aligned}
& R(\omega)=|\sqrt{2 \kappa} E(\omega)-1|^{2}, \\
& T(\omega)=|\sqrt{2 \kappa} E(\omega)|^{2}, \quad S^{v}(\omega)=2|\sqrt{2 \kappa} F(\omega)|^{2}, \\
& S_{1}(\omega)=\gamma_{1}\left|V_{1}(\omega)\right|^{2}, \quad S_{2}(\omega)=\gamma_{2}\left|V_{1}(\omega)\right|^{2} .
\end{aligned}
$$

In Eqs. (14), $R(\omega)$ and $T(\omega)$ are the contributions arising from the presence of a single photon in the input field. $S^{v}(\omega)$ is contribution the nature of the vacuum field. $S_{1}(\omega)$ and $S_{2}(\omega)$ are the contributions from the fluctuation of the effective of the mechanical oscillator mode. Equations (14) are similar to the output field in Ref. [16]. However, our results are different from the results in Ref. [16]. For example, the BEC acts as the vacuum state of the mechanical oscillator mode. Also, the nanomechanical mirror is replaced by a cigar-shaped BEC.

\section{Results and discussion}

In this section we discuss a quantum router based on the numerical solutions of Eqs. (14). To make this study of the quantum router in the BEC-optomechanical experimentally feasible, we choose the realistic parameters from presently available experimental setups [25-27]. The parameters of the systems are as follows: the wavelength of the cavity field is $\lambda=780 \mathrm{~nm}$ and the cavity length is $L=1.25 \times 10^{-4}$. We consider $N=1.2 \times 10^{5}$ atoms trapped inside the optomechanical cavity with a recoil of $\omega_{\text {res }}=2 \pi \times 3.8 \mathrm{kHz}$. The maximum coupling strength $g_{0}=2 \pi \times 10.9 \mathrm{MHz}$ and the detuning $\Delta_{a}=2 \pi \times 32 \mathrm{GHz}$ and $\mathrm{BEC}$ acts as the mechanical oscillator mode with a damping rate $\gamma_{s m}=2 \pi \times 0.4 \mathrm{kHz}$. The cavity with decay rate is $\kappa=2 \pi \times 1.3 \mathrm{kHz}$.

In this paper, we consider the following two conditions. First, the optomechanical system is operated in resolved sideband condition $\kappa \ll \omega_{m}$. Second, we will assume $\Delta=\omega_{m}$, and anti-Stokes scattering survives inside the cavity. The reflection spectrum $R(\omega)$ and the transmission spectrum $T(\omega)$ of the single photon as a function of normalized frequency $\omega / \omega_{m}$ are discussed in Figs. 2 and 3. One can find from Fig. 2 that only an inverted Lorentzian is in the reflection spectrum in the absence in the pumping field. However, the situation is completely different when we increase the pumping field. On one hand, the transparency window appears with the increase of the pumping field, on the other hand, the width of the transparency window increases with increase of the pumping field. One can find from Fig. 3 that only a standard Lorentzian appears in the transmission spectrum in the absence of the pumping field. In the presence 


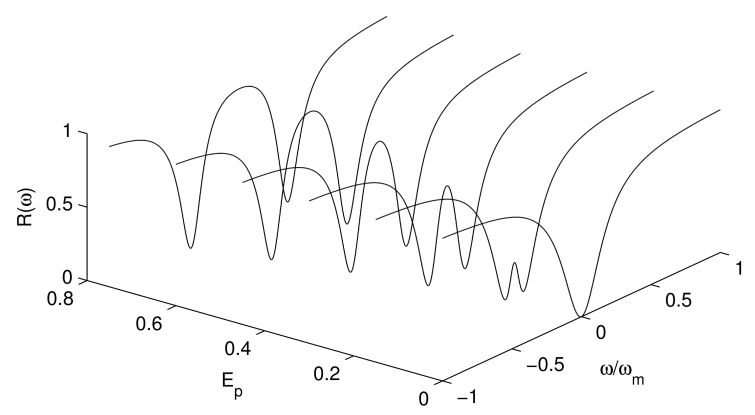

Fig. 2. The reflection spectrum $R(\omega)$ of the single photon as a function of the normalized frequency $\omega / \omega_{m}$, and the normalized field strength $E_{p}$ (units of $E_{0}=$ $2.0 \times 10^{3} \mathrm{~Hz}$ ). The other parameters are $\lambda=780 \mathrm{~nm}$, $L=1.25 \times 10^{-4}, N=1.2 \times 10^{5}, \omega_{\text {res }}=2 \pi \times 3.8 \mathrm{kHz}$, $\gamma_{s m}=2 \pi \times 0.21 \mathrm{kHz}, \kappa=2 \pi \times 1.3 \mathrm{kHz}, \Delta_{a}=$ $2 \pi \times 32 \mathrm{GHz}$ and $g_{0}=2 \pi \times 10.9 \mathrm{MHz}$.

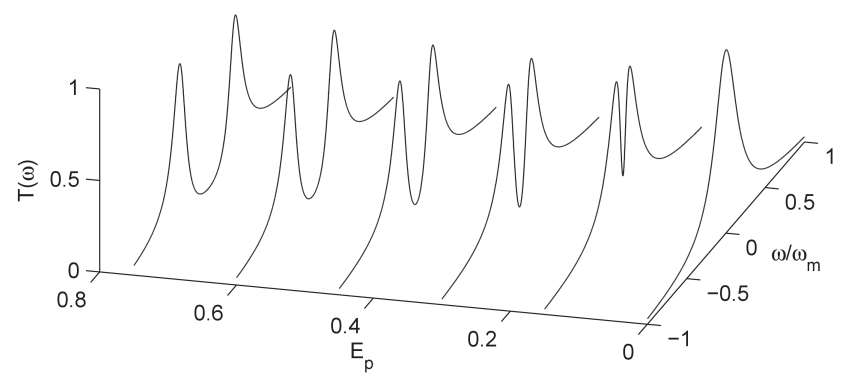

Fig. 3. The transmission spectrum $T(\omega)$ of the single photon as a function of the normalized frequency $\omega / \omega_{m}$, and the normalized field strength $E_{P}$. The other parameters are the same as those shown in Fig. 2.

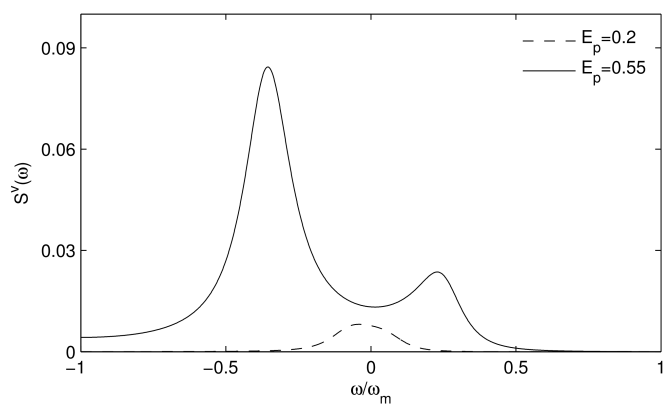

Fig. 4. The noise spectrum $S^{v}(\omega)$ as a function of the normalized frequency $\omega / \omega_{m}$. The other parameters are the same as those shown in Fig. 2.

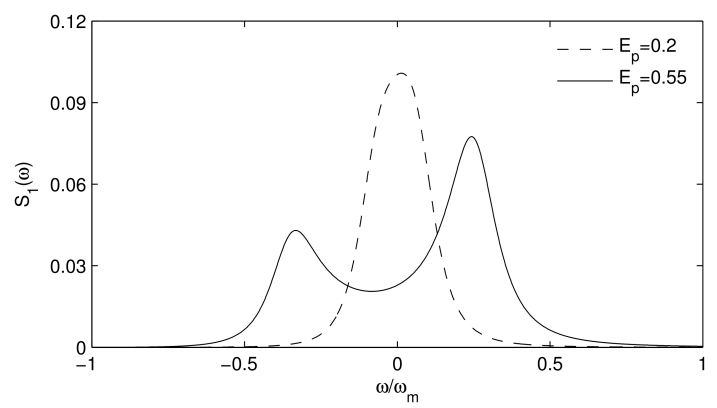

Fig. 5. The noise spectrum $S_{1}(\omega)$ as a function of the normalized frequency $\omega / \omega_{m}$. The other parameters are the same as those shown in Fig. 2.

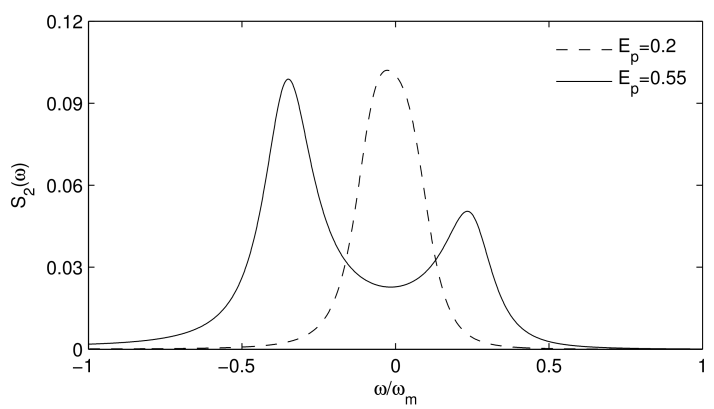

Fig. 6. The noise spectrum $S_{2}(\omega)$ as a function of the normalized frequency $\omega / \omega_{m}$. The other parameters are the same as those shown in Fig. 2.

of the pumping field, the situation is completely different. Generally speaking, in the absence of the pumping field, the output field can transmit completely from the right port, while the reflection is totally suppressed. We note that $R\left(\omega_{m}\right) \approx 0$ and $T\left(\omega_{m}\right) \approx 1$, so the single photon is completely reflected through the cavity to the left output port. However, in Figs. 2 and 3, the situation is completely different for an increase of the pumping field. The output field can completely reflect from the left port but its transmission is completely suppressed.

We now discuss the effect of the quantum noise on the reflection and transmission spectrum of a single-photon. From Fig. 4, we can see the behavior of the vacuum noise $S^{v}(\omega)$ for two different values of the field strength. The contribution of the noises maximum is about $10 \%$, which is significant. For the thermal noise due to the effective mirror $S_{1}(\omega)$ and $S_{2}(\omega)$ for two different values of the field strength are shown in Figs. 5 and 6. One can find that for larger pumping field powers, $S_{1}(\omega)$ and $S_{2}(\omega)$ split into two separate peaks. The contribution of the thermal noises maximum is about $10 \%$, which is significant, too.

\section{Conclusion}

We discuss the single-photon quantum router in a BEC-optomechanical system, by following the same method as reported in Ref. [16]. The system consists of a cigar-shaped BEC trapped inside an optical cavity. We have shown that the BEC-optomechanical system can be used as a single-photon quantum router. We have also shown that the quantum noise sources for this phenomenon are very small. In addition, we have suggested a specific set of experimental parameters to be observed in a laboratory.

\section{Acknowledgments}

Project supported by the National Natural Science Foundation of China (Grant No. 11564034, and Grant No. 11105062), the scientific research funds of College of Electrical Engineering, Northwest University for Nationalities (No. xbmuyjrc201115). 


\section{References}

[1] H.J. Kimble, Nature 453, 1023 (2008).

[2] S. Perseguers, M. Lewenstein, A. Acin, J.I. Cirac, Nat. Phys. 6, 539 (2010).

[3] W. Tittel, J. Brendel, H. Zbinden, N. Gisin, Phys. Rev. Lett. 84, 4737 (2000).

[4] L.M. Duan, M.D. Lukin, J.I. Cirac, P. Zoller, Nature 414, 413 (2001).

[5] S. Ritter, C. Nölleke, C. Hahn, A. Reiserer, A. Neuzner, M. Uphoff, M. Mücke, E. Figueroa, J. Bochmann, G. Rempe, Nature 484, 195 (2012).

[6] C.H. Bennett, Phys. Rev. Lett. 68, 3121 (1992).

[7] B. Yurke, J.S. Denker, Phys. Rev. A 29, 1419 (1984).

[8] L. Zhou, Z.R. Gong, Y.X. Liu, C.P. Sun, F. Nori, Phys. Rev. Lett. 101, 100501 (2008).

[9] K. Xia, J. Twamley, Phys. Rev. X 3, 031013 (2013).

[10] L. Zhou, L.P. Yang, Y. Li, C.P. Sun, Phys. Rev. Lett. 111, 103604 (2013).

[11] I. Shomroni, S. Rosenblum, Y. Lovsky, O. Bechler, G. Guendelman, B. Dayan, Science 345, 903 (2014).

[12] J. Lu, L. Zhou, L.M. Kuang, F. Nori, Phys. Rev. A 89, 013805 (2014).

[13] W.B. Yan, H. Fan, Sci. Rep. 4, 4820 (2014).

[14] T. Aoki, A.S. Parkins, D.J. Alton, C.A. Regal, Barak Dayan, E. Ostby, K.J. Vahala, H.J. Kimble, Phys. Rev. Lett. 102, 083601 (2009).

[15] I.C. Hoi, C.M. Wilson, G. Johansson, T. Palomaki, B. Peropadre, P. Delsing, Phys. Rev. Lett. 107, 073601 (2011).

[16] G.S. Agarwal, S. Huang, Phys. Rev. A 85, 021801(R) (2012).

[17] M. Mücke, E. Figueroa, J. Bochmann, C. Hahn, K. Murr, S. Ritter, C.J. Villas-Boas, G. Rempe, $\mathrm{Na}$ ture 465, 755 (2010).
[18] P. Longo, P. Schmitteckert, K. Busch, Phys. Rev. Lett. 104, 023602 (2010).

[19] P. Bermel, A. Rodriguez, S.G. Johnson, J.D. Joannopoulos, M. Soljačić, Phys. Rev. A 74, 043818 (2006).

[20] D.E. Chang, A.S. Srensen, E.A. Demler, M.D. Lukin, Nat. Phys. 3, 807 (2007).

[21] X.M. Li, L.F. Wei, Phys. Rev. A 92, 063836 (2015).

[22] B. Chen, J.J. Li, C. Jiang, K.D. Zhu, Phot. Technol. Lett. IEEE 24, 766 (2012).

[23] C. Jiang, B. Chen, K.D. Zhu, J. Appl. Phys. 112 033113 (2012).

[24] T.J. Kippenberg, K.J. Vahala, Science 321, 1172 (2008).

[25] F. Brennecke, T. Donner, S. Ritter, T. Bourdel, M.L. Köhl, T. Esslinger, Nature 450, 268 (2007)

[26] Y. Colombe, T. Steinmetz, G. Dubois, F. Linke, D. Hunger, J. Reichel, Nature 450, 272 (2007).

[27] F. Brennecke, S. Ritter, T. Donner, T. Esslinger, Science 322, 235 (2008).

[28] K.A. Yasir, W.M. Liu, Sci. Rep. 5, 10612 (2015).

[29] K.A. Yasir, W.M. Liu, Sci. Rep. 6, 22651 (2016).

[30] K. Zhang, W. Chen, M. Bhattacharya, P. Meystre, Phys. Rev. A 81, 013802 (2010).

[31] Y.J. Wang, D.Z. Anderson, V.M. Bright, E.A. Cornell, Q. Diot, T. Kishimoto, M. Prentiss, R.A. Saravanan, S.R. Segal, S.J. Wu, Phys. Rev. Lett. 94, 090405 (2005).

[32] P. Treutlein, D. Hunger, S. Camerer, T.W. Hänsch, J. Reichel, Phys. Rev. Lett. 99, 140403 (2007).

[33] J. Fortágh, C. Zimmermann, Rev. Mod. Phys. 79, 235 (2007).

[34] C.W. Gardiner, P. Zoller, Quantum Noise, Springer, Berlin 2000.

[35] D.F. Walls, G.J. Milburn, Quantum Optics, Springer, Berlin 1994. 